\title{
Prevention of atherosclerosis and drug treatment of high-risk lipid abnormalities in children and adolescents
}

\author{
Prevenção da aterosclerose e tratamento medicamentoso de anormalidades \\ lipídicas de alto risco em crianças e adolescentes \\ Priscilla Severino Gonçalves de Castro' ${ }^{1}$, Fernanda Luisa Ceragioli Oliveira ${ }^{2}$
}

\section{Resumo}

Objetivo: Discutir os fatores de risco da aterosclerose na pediatria e as recomendações de dieta e exercício físico e, principalmente, o tratamento medicamentoso de anormalidades lipídicas de alto risco.

Fontes dos dados: Os dados foram obtidos por meio de artigos indexados na MEDLINE, publicados nos últimos 5 anos.

Síntese dos dados: Crianças com dislipidemia grave ou outros fatores de risco, como história familiar de doença cardiovascular precoce ou outros sinais de síndrome metabólica, podem necessitar de tratamento com drogas hipolipemiantes. Novas recomendações do consenso americano indicam tratamento medicamentoso antes dos 10 anos de idade, dependendo da magnitude de outros fatores de risco para doença cardiovascular. Cabe aos pediatras saber quando diagnosticar dislipidemia, quando indicar o tratamento medicamentoso e quais as drogas que podem ser utilizadas em crianças e adolescentes com menor risco e prejuízo ao seu desenvolvimento.

Conclusões: O tratamento da dislipidemia deve ser inicialmente realizado sempre por meio de mudanças nos hábitos de vida, dando-se ênfase à prevenção. Crianças com anormalidades lipídicas de alto risco merecem tratamento medicamentoso. A decisão a ser tomada junto com os pais sempre deve respeitar os riscos e os benefícios que o tratamento acarretará ao paciente.

J Pediatr (Rio J). 2009;85(1):6-14: Aterosclerose, hipercolesterolemia, lipídeos, medicamento, pediatria.

\section{Introdução}

A doença aterosclerótica inicia silenciosamente na infância, progride durante a adolescência e a idade adulta e acarreta doença cardiovascular em adultos ou idosos ${ }^{1-3}$. A doença foi precocemente evidenciada em fetos filhos de mães com hipercolesterolemia ${ }^{1}$. Sua patogênese envolve o sistema inflamatório e imunológico. A lipoproteína de baixa densidade de colesterol (LDL-C) é oxidada e acumulada abaixo do

\section{Abstract}

Objective: To discuss risk factors of atherosclerosis in pediatrics, dietary and physical activity guidelines, and, mainly, drug treatment of high-risk lipid abnormalities.

Sources : Data were obtained from articles indexed in MEDLINE, published over the last 5 years.

Summary of the findings: Children with severe dyslipidemia or additional risk factors such as family history of early cardiovascular disease or other signs of metabolic syndrome may need treatment with hypolipidemic drugs. New recommendations from the U.S. guidelines indicate drug treatment before the age of 10 years according to the magnitude of the additional risk factors for cardiovascular disease. Pediatricians should know when to diagnose dyslipidemia, when to indicate drug treatment and which medication can be used in children and adolescents with the least risk or harm to their development.

Conclusions: The first-line treatment of dyslipidemia consists of lifestyle changes, focusing on prevention. Children with high-risk lipid abnormalities should be considered for drug treatment. Decisions to be made together with the parents must be evaluated taking into consideration risks and benefits of the medication to the patient.

J Pediatr (Rio J). 2009;85(1):6-14: Atherosclerosis, hypercholesterolemia, lipids, medication, pediatrics.

endotélio vascular, resultando em depósitos filiformes de gordura na íntima vascular que vai ser coberta por uma capa fibrosa (placa). Esta, dependendo de sua estabilidade, pode causar eventos isquêmicos e trombóticos, como a doença coronariana e o infarto agudo do miocárdio, respectivamente ${ }^{4}$.

Os estudos em aterosclerose iniciaram por meio de autópsias em indivíduos jovens que morreram durante combate.

1. Médica pediatra. Especialista em Nutrologia Pediátrica, Universidade Federal de São Paulo - Escola Paulista de Medicina (UNIFESP-EPM), São Paulo, SP. 2. Doutora. Médica pediatra. Responsável, Setor de Suporte Nutricional, Disciplina de Nutrologia, Departamento de Pediatria, UNIFESP-EPM, São Paulo, SP. Não foram declarados conflitos de interesse associados à publicação deste artigo.

Como citar este artigo: de Castro PS, Oliveira FL. Prevention of atherosclerosis and drug treatment of high-risk lipid abnormalities in children and adolescents. J Pediatr (Rio J). 2009;85(1):6-14.

Artigo submetido em 28.05.08, aceito em 06.08.08.

doi:10.2223/JPED.1852 
Tabela 1 - Perfil lipídico para crianças acima de 2 anos e adolescentes, segundo a I Diretriz de Prevenção da Aterosclerose na Infância e na Adolescência ${ }^{13}$

\begin{tabular}{lccc}
\hline Lipoproteínas $(\mathbf{m g} / \mathbf{d L})$ & Desejáveis & Limítrofes & Aumentados \\
\hline CT & $<150$ & $150-169$ & $>170$ \\
LDL-C & $<100$ & $100-129$ & $\geq 130$ \\
HDL-C & $\geq 45$ & - & - \\
TG & $<100$ & $100-129$ & $\geq 130$ \\
\hline
\end{tabular}

$\overline{\mathrm{CT}}=$ colesterol total; HDL-C = fração do colesterol de lipoproteína de alta densidade; LDL-C = fração do colesterol de lipoproteína de baixa densidade; TG $=$ triglicérides.

Em indivíduos com idade média de 22 anos, foi observado que 45\% tinham evidência de aterosclerose coronariana e 5\% em estágio grave ${ }^{2}$. Estudos de patologia e de epidemiologia correlacionam à doença aterosclerótica os seguintes fatores de risco: anormalidades no perfil lipídico, hipertensão arterial, hiperglicemia e obesidade ${ }^{5-9}$.

Estudo envolvendo adolescentes mostra que fatores de risco modificáveis, como idade, sexo, níveis de LDL-C, lipoproteína de alta densidade de colesterol (HDL-C), triglicérides, tabagismo, pressão arterial e obesidade, estão relacionados às lesões ateroscleróticas nas artérias coronarianas e aorta em diferentes graus. Esses fatores de risco tendem a se tornar cada vez mais importantes, pois tendem a persistir e, tipicamente, piorar com o avançar da idade ${ }^{1}$.

Raitakari et al. ${ }^{6}$ realizaram seguimento de 2.229 adultos jovens, entre 24 e 49 anos, que haviam participado previamente do estudo dos fatores de risco para aterosclerose na infância, com idade entre 3 e 9 anos. Analisaram-se níveis de LDL-C, pressão arterial sistólica e tabagismo relacionando esses fatores de risco à espessura da camada íntima-média da artéria carótica, obtida por meio da ultrassonografia realizada na idade adulta. Foi realizada análise de regressão linear, correção para idade, sexo e fatores de risco vigentes, sendo encontrada forte associação entre os fatores de risco no período da adolescência e o aumento da camada íntima-média na idade adulta ${ }^{6}$. Os fatores de risco encontrados na adolescência têm maior valor preditivo daqueles analisados no momento do exame de imagem na idade adulta, enfatizando a importância dos fatores de risco em idades precoces ${ }^{6-9}$. Como importante fator de risco, destaca-se a história familiar de hipercolesterolemia e de doença cardiovascular, que está diretamente relacionada com os níveis de lipoproteínas encontrados nas crianças ${ }^{10}$. Estudo brasileiro identificou que dividindo as crianças obesas por tercil de modelo homeostático de resistência à insulina (HOMA-IR - homeostasis model of insulin resistance) , 41,2\% dos pacientes no primeiro tercil apresentava mais de dois fatores de risco para doença cardiovascular $^{11}$.

O aumento nos níveis de LDL-C pode ser secundário aos inadequados hábitos alimentares, ao estilo de vida e/ou pode ser resultado de alterações do metabolismo lipídico decorrentes de fatores genéticos. A principal representante na infância e na adolescência de alteração no metabolismo lipídico é a hipercolesterolemia familiar. Caracteriza-se por níveis elevados de colesterol total e LDL-C, história familiar de hipercolesterolemia e por morte prematura decorrente de doença cardiovascular em parentes de primeiro grau ${ }^{12}$.

Com aumento da prevalência de obesidade infantil e consequente aumento de risco de eventos cardiovasculares em população cada vez mais jovem, nova discussão a respeito do tratamento medicamentoso nessa população merece especial atenção.

\section{Anormalidades lipídicas}

Atualmente, para crianças acima de 2 anos de idade, os valores de normalidade do perfil lipídico adotados pela Sociedade Brasileira de Pediatria e Sociedade Brasileira de Cardiologia estão descritos na Tabela 1. As dosagens séricas de colesterol e frações devem ser realizadas após jejum de 12 horas.

O primeiro consenso pediátrico foi publicado em 1992, pelo National Cholesterol Education Program (NCEP) ${ }^{14}$, e cita duas formas de detectar dislipidemias em crianças e adolescentes. Uma delas é que crianças com história familiar de doença cardiovascular precoce devem dosar os níveis séricos de colesterol total e frações. História familiar é definida como pais ou avós com idade inferior a 55 anos com evidência de aterosclerose: doença vascular periférica, doença cerebrovascular, submetidos a procedimento em artéria coronariana, vítima de infarto agudo do miocárdio ou morte súbita cardíaca. A outra é que crianças com história de hipercolesterolemia apenas nos pais (nível de colesterol total $\geq 240$ $\mathrm{mg} / \mathrm{dL}$ ) devem dosar seu nível de colesterol total. Se o nível de colesterol for limítrofe (entre 170 e 200 mg/dL), a dosagem de colesterol deve ser repetida. Se houver duas medidas de colesterol total $\geq 170 \mathrm{mg} / \mathrm{dL}$ ou a primeira medida for $\geq$ $200 \mathrm{mg} / \mathrm{dL}$, a criança deve ter as frações de colesterol séricas mensuradas. Devido à variação individual, as dosagens séricas de colesterol e frações devem ser realizadas por duas vezes no mesmo laboratório de análise clínica, para confirmação do diagnóstico de dislipidemia. 
Em 2005, no Brasil, foi criada pela Sociedade Brasileira de Cardiologia e outras sociedades civis, como a Sociedade Brasileira de Pediatria, a I Diretriz de Prevenção da Aterosclerose na Infância e na Adolescência ${ }^{13}$. A recomendação para análise do perfil lipídico faz-se em crianças: 1 ) cujos pais ou avós têm história de aterosclerose com idade inferior a 55 anos; 2 ) cujos pais apresentam colesterol total $\geq 240 \mathrm{mg} / \mathrm{dL}$; 3) que apresentam outros fatores de risco como hipertensão arterial, obesidade, tabagismo ou consumo de dieta rica em gorduras saturadas e/ou trans; 4) que utilizam corticosteroides ou são portadoras de doenças que cursam com dislipidemia (síndrome da imunodeficiência adquirida, SIDA ou AIDS, hipotireoidismo, lúpus, doenças renais crônicas, anorexia nervosa); e 5) que possuem manifestações clínicas de dislipidemias (xantomas, xantelasmas, arco corneal, dores abdominais recorrentes, pancreatites). Nessa diretriz, orienta-se a coleta do perfil lipídico (triglicérides, colesterol total, LDL-C, lipoproteína de densidade muito baixa de colesterol, VLDL-C e HDL-C) em todas as crianças com as características acima citadas, após 12 horas de jejum, em laboratório clínico confiável ${ }^{14}$.

As recomendações para reavaliação e tratamento restringem àquelas com LDL-C limítrofes (110 a 129 mg/dL) ou elevado $(\geq 130 \mathrm{mg} / \mathrm{dL})^{13,15}$.

\section{Recomendações dietéticas}

\section{Prevenção}

A abordagem populacional nos consensos recomenda que as crianças com idade superior a 2 anos adotem dieta restrita em gordura e colesterol apropriada para manter o crescimento e desenvolvimento, além de manter peso corporal "desejável". A recomendação diária para a população geral consiste em consumir $300 \mathrm{mg} / \mathrm{dL}$ de colesterol e no máximo $30 \%$ do valor energético total em gordura, sendo permitido que $10 \%$ desse valor seja sob a forma de gordura saturada. Atualmente, existe restrição para ingestão de gordura trans, que está presente na gordura hidrogenada usada no preparo de produtos industrializados ${ }^{16}$. Seu consumo está diretamente relacionado com aumento da concentração de LDL-C de pequeno tamanho, que apresenta risco aterogênico maior que o LDL-C de maior tamanho ${ }^{16}$. A American Heart Association (AHA) recomenda que o teor de gordura trans na alimentação seja de até $1 \%$ do valor energético total de lipídeos ${ }^{16}$.

Recomendação dietética inclui consumo de peixes ricos em ômega 3, duas porções por semana, pois está associado com redução do risco de morte súbita e morte por doença coronariana em adultos ${ }^{16}$.

\section{Tratamento}

As recomendações dietéticas do NCEP ${ }^{14}$ para crianças com LDL-C elevado são: ingestão de colesterol diário de $200 \mathrm{mg} / \mathrm{dL}$ e gordura saturada na quantidade máxima de $7 \%$ do valor total de energia ${ }^{13}$. A quantidade de gordura trans ingerida deve ser idêntica à utilizada na população em geral.
Estudo duplo cego, randomizado, placebo controlado de ácido docosahexanoico (ômega 3) em 20 crianças com hiperlipidemia mostrou mudanças significativas e favoráveis na distribuição das subclasses das lipoproteínas, com aumento das lipoproteínas maiores e diminuição das menores e mais aterogênicas ${ }^{17}$. Crianças com hipertrigliceridemia beneficiam-se com o consumo de ômega 3 , e a suplementação medicamentosa deve ser considerada se a quantidade terapêutica não for atingida.

\section{Recomendações de atividade física}

O tempo diante da televisão, videogame e computador deve ser limitado a $2 \mathrm{~h} /$ dia na prevenção da aterosclerose, além da necessidade de se estimular a atividade física, realizada de forma prazerosa por, no mínimo, $30 \mathrm{~min} / \mathrm{dia}$, de 3 a 4 vezes por semana ${ }^{14}$. Estudo brasileiro encontrou prevalência de sedentarismo em mais de $70 \%$ das crianças e adolescentes com excesso de peso ${ }^{11}$.

A atividade física parece atuar no perfil lipídico, porém há grande dificuldade metodológica em se quantificar atividade física em crianças e adolescentes. A atividade aeróbica parece estar relacionada com diminuição nos níveis de triglicérides, colesterol total e LDL-C, assim como aumento nos níveis de HDL-C ${ }^{2,18,19}$. Embora essas alterações ocorram de forma modesta, a atividade física tem outros benefícios como diminuição do índice de massa corpórea, da pressão arterial e, consequentemente, diminuição da doença cardiovascular $^{19,20}$.

\section{Tratamento medicamentoso da dislipidemia}

\section{Consensos}

O NCEP ${ }^{14}$ recomendava tratamento medicamentoso para crianças maiores de 10 anos de idade com LDL-C $\geq 190 \mathrm{mg} / \mathrm{dL}$, cujos níveis de colesterol permanecerem elevados apesar das medidas dietéticas durante o período de 6 a 12 meses. Considerava, também, o tratamento àquelas com LDL-C $\geq 160$ $\mathrm{mg} / \mathrm{dL}$ com presença de dois ou mais fatores de risco para doença cardiovascular ou história familiar de doença cardiovascular precoce. A droga de primeira escolha constituiu as resinas biliares por não serem absorvidas ${ }^{13}$.

Segundo a diretriz brasileira ${ }^{13}$, todas as crianças com LDL-C > $130 \mathrm{mg} / \mathrm{dL}$ devem ser acompanhadas. A primeira opção deve ser sempre o tratamento dietético. O uso de fármacos fica restrito para as crianças com idade acima de 10 anos que apresentam níveis de LDL-C persistentemente elevados, a despeito da orientação nutricional. Os valores de referência do LDL-C para a intervenção com hipolipemiantes dependem dos fatores de risco presentes, como história familiar e magnitude da elevação do LDL-C. O tratamento farmacológico tem sido indicado preferencialmente para as situações de maior risco e de falha em modificações do estilo de vida para se atingir o nível ideal de LDL-C $(<110 \mathrm{mg} / \mathrm{dL})$ ou aceitável (110 a $130 \mathrm{mg} / \mathrm{dL})^{14}$. 


\section{Estudos clínicos \\ Dislipidemia primária}

Estudos de intervenção em crianças com dislipidemia primária foram importantes para demonstrar a segurança medicamentosa, o não-comprometimento do crescimento e do desenvolvimento e, principalmente, a reversão das anormalidades vasculares funcionais com a instituição do tratamento medicamentoso. Dirisamer et al. ${ }^{12}$ realizaram estudo com 20 adolescentes, entre 10 e 17 anos, com diagnóstico de hipercolesterolemia familiar em uso de sinvastatina por 1 ano. Os níveis de LDL-C reduziram em 30\% naqueles tratados com dose de $5 \mathrm{mg} / \mathrm{dia}, 30 \%$ naquelas que receberam $10 \mathrm{mg} / \mathrm{dia}$ e, finalmente, $36 \%$ naquelas que receberam a dose de 20 $\mathrm{mg} /$ dia. Dois pacientes tiveram elevação da enzima creatinoquinase e uma elevação das transaminases (alanino aminotransferase e aspartato aminotransferase). Os efeitos colaterais apareceram nas primeiras 4 semanas de tratamento e desapareceram até o sétimo dia, e nenhum paciente teve que cessar o tratamento devido aos efeitos colaterais ${ }^{10}$.

Estudo randomizado, duplo cego, placebo controlado com seguimento de 2 anos, em pacientes com idade entre 8 e 18 anos, com diagnóstico de hipercolesterolemia familiar, utilizando dose de $20 \mathrm{mg}$ para menores de 14 anos e $40 \mathrm{mg}$ nos maiores de 14 anos, demonstrou queda nos níveis de LDL-C de $24,1 \%$. Quanto à segurança medicamentosa, foram analisados nível de educação, crescimento e desenvolvimento, assim como estadiamento puberal e função endócrina, sem encontrar diferença significativa entre os grupos de intervenção e controle. $\mathrm{O}$ achado mais importante deste estudo foi a diminuição na espessura da camada íntima-média nos pacientes que receberam pravastatina, identificando que as lesões ateroscleróticas dessas crianças foram reversíveis ${ }^{21}$.

Outro estudo prospectivo com 35 crianças entre 4,1 e 18,5 anos com hipercolesterolemia familiar, em uso de pravastatina, foi obtida redução de $33 \%$ nos níveis de LDL-C após 1 ano de seguimento com doses de 10 a $60 \mathrm{mg}$. O uso da pravastatina mostrou-se seguro e bem tolerado mesmo nos pacientes abaixo de 8 anos de idade e não se verificou alterações no crescimento, atraso no desenvolvimento puberal ou modificações nos níveis de estradiol ou testoterona dos pacientes $^{22}$.

Foram randomizadas 54 adolescentes do sexo feminino com hipercolesterolemia familiar em estudo multicêntrico e placebo controlado, com uso da dose inicial de 20 mg de lovastatina por 4 semanas com progressão para $40 \mathrm{mg}$ por mais 20 semanas, totalizando 24 semanas de seguimento. A lovastatina foi bem tolerada, e a média de redução no nível do LDL-C foi de $27 \%{ }^{23}$.

Outro estudo randomizado, duplo cego, placebo controlado, com 186 pacientes com hipercolesterolemia familiar e idade entre 8 e 18 anos, foi realizado com uso de pravastatina durante média de 4,5 anos na dose de 20 mg e 40 mg, respectivamente para menores e maiores de 14 anos. Os níveis de LDL-C diminuíram em 29,2\% e houve diminuição da camada íntima-média nos pacientes que receberam pravastatina ${ }^{24}$. Assim, parece cientificamente seguro o uso de estatinas nas dislipidemias primárias, avaliando-se sempre o custo e o benefício de sua utilização no paciente jovem.

\section{Dislipidemia secundária}

A síndrome metabólica (SM) é distúrbio nutricional presente nos países em desenvolvimento e desenvolvidos com alta prevalência na população adulta e de crescente prevalência na população pediátrica ${ }^{25-27}$. Identificou-se prevalência de SM em adolescentes americanos em $6,8 \%$ de sobrepeso e em $28,7 \%$ de obesos ${ }^{28}$. Estudo brasileiro em crianças obesas demonstrou prevalência de SM em 17,3\% das crianças obesas com idade entre 7 e 10 anos $^{11}$.

A SM é transtorno complexo representado por um conjunto de fatores de risco cardiovascular usualmente relacionados à deposição central de gordura e à resistência insulínica. Os componentes da doença encontrados em adultos são: obesidade abdominal, hipertrigliceridemia, diminuição nos níveis séricos de HDL-C, aumento da pressão arterial e aumento da glicemia de jejum ${ }^{29}$. A obesidade constitui fator de risco grave para o desenvolvimento de dislipidemia. Embora não haja critérios definidos para o diagnóstico dessa síndrome em crianças e adolescentes, os valores de glicose, resistência insulínica, triglicérides, proteína C-reativa, interleucina-6 e pressão arterial sistólica aumentam significativamente com aumento da obesidade, assim como os níveis séricos de HDL-C e adiponectina diminuem ${ }^{30}$. A SM está associada com aumento do desenvolvimento de doenças metabólicas e cardiovasculares, como aterosclerose e diabetes melito tipo $2^{31-33}$

Estudo de seguimento por um período de 25 a 30 anos foi realizado com 814 crianças, com idade inicial entre 5 e 19 anos. Demonstrou-se que 3,9\% das crianças apresentaram prevalência de SM na infância, das quais $69 \%$ tinham SM persistente durante todo período do estudo, em comparação com $24 \%$ daquelas que não tinham SM na infância ${ }^{32}$.

O Bogalusa Heart Study demonstrou que crianças obesas em idade escolar têm entre 2,4 a 7,1 vezes mais chance de ter colesterol total elevado, LDL-C e triglicérides e 12,6 vezes mais chance de ter hiperinsulinemia ${ }^{5}$. Estudo brasileiro encontrou em crianças e adolescentes com excesso de peso risco 2,8 vezes maior de desenvolverem dislipidemia ${ }^{34}$.

Sun et al. ${ }^{35}$ demonstraram que crianças com aumento da pressão arterial têm maior risco de desenvolver hipertensão arterial sistêmica na idade adulta, assim como SM. Recentemente, o risco de SM pôde ser identificado na primeira década de vida por meio do índice de massa corporal e da circunferência abdominal mensurados precocemente aos 6 anos de idade $^{36}$.

Estudos com tratamento medicamentoso em crianças com SM são raros na literatura. Estudo híbrido, duplo cego, placebo controlado, multicêntrico e randomizado realizou terapia medicamentosa com atorvastatina em 187 crianças entre 
10 e 17 anos de idade, com diagnóstico de dislipidemia primária ou hipercolesterolemia grave (definida como LDL-C $\geq$ $190 \mathrm{mg} / \mathrm{dL}$ ). Entre os pacientes que receberam atorvastatina, $60 \%$ atingiram níveis de LDL-C $<130 \mathrm{mg} / \mathrm{dL}$, enquanto no grupo controle não houve paciente que atingiu esse valor de LDL-C após 6 meses de seguimento. Os pacientes tiveram boa tolerância e não houve efeitos no crescimento ou desenvolvimento ${ }^{37}$.

Existem indivíduos que são considerados de alto risco para o desenvolvimento de anormalidades lipídicas. Essas condições incluem: diabetes melito, doença renal crônica, transplante cardíaco, doença de Kawasaki, doença cardíaca congênita, doença inflamatória crônica, câncer e AIDS ${ }^{38}$.

O diabetes tipo 1 é doença metabólica caracterizada por defeitos na secreção de insulina e a hiperglicemia está associada como fator primário no desenvolvimento de aterosclerose. Doença renal crônica, causa anormalidades cardíacas como pericardite, arritmias e disfunção ventricular, que, somadas à uremia e hipertensão arterial, vão aumentar o risco do desenvolvimento de aterosclerose. Crianças transplantadas cardíacas têm algum grau de aterosclerose nas artérias coronarianas ainda no primeiro ano após o transplante. Outra doença que causa alterações coronarianas, a doença de Kawasaki, também predispõe as crianças à doença aterosclerótica precoce. Lupus eritematoso sistêmico e artrite reumatoide são doenças inflamatórias crônicas que têm associação com aterosclerose devido à própria inflamação (parte da fisiopatologia da doença aterosclerótica), assim como a corticoterapia usada no seu tratamento. As doenças cardíacas congênitas que causam anormalidades coronarianas e lesões obstrutivas estão também relacionadas com aterosclerose precoce. As crianças sobreviventes de câncer têm menor massa ventricular, cursam com diminuição nos níveis de hormônio do crescimento, causando desenvolvimento de obesidade, resistência insulínica, diabetes melito tipo 2 , com consequente $\mathrm{SM}^{38,39}$.

Outro grupo de risco para doença aterosclerótica precoce são as crianças infectadas pelo HIV que estão em uso de inibidores da protease. Estudos transversais mostram associação dessas drogas com hipercolesterolemia e hipertrigliceridemia ${ }^{40}$. Estudos que examinaram a incidência de hipercolesterolemia em crianças em tratamento com inibidores da protease observaram aumento no colesterol durante todo o tratamento ${ }^{41}$. No primeiro estudo longitudinal prospectivo realizado para examinar o efeito dos inibidores da protease na população pediátrica infectadas pelo HIV, em adição aos 13\% que possuíam alteração do perfil lipídico, $13 \%$ desenvolveram hipercolesterolemia com o tratamento ${ }^{42}$.

Manual para tratamento da dislipidemia secundária à AIDS em adultos preconiza pravastatina ou atorvastatina para pacientes com triglicérides entre 200 e 500 mg/dL e gemfibrozil àqueles com triglicérides $>500 \mathrm{mg} / \mathrm{dL}$. Sinvastatina e lovastatina devem ser evitadas, devido à interação medicamentosa com o esquema antirretroviral ${ }^{43}$. Estudos prospectivos mostraram eficácia e segurança no uso das estatinas: pravastatina, atorvastatina, sinvastatina e fluvastatina e dos fibratos: benzofibrato, gemfibrozil, fenofibrato e ciprofibrato, para pacientes em esquema antirretroviral, sem alterações nos níveis de cluster of differentiation 4, carga viral, creatinoquinase e transaminases, sugerindo que não há interação com a terapia antirretroviral ${ }^{44,45}$.

Diante do exposto, torna-se claro que a prevenção da aterosclerose e suas repercussões no sistema cardiovascular devem iniciar ainda na infância. Parece que o tratamento dietético em casos graves não é suficiente para cessar o desenvolvimento da doença, ficando evidente a necessidade de terapia medicamentosa associada à dietética para abortar a continuidade do processo.

\section{Novas recomendações para tratamento medicamentoso em crianças e adolescentes com dislipidemia}

Existem teorias de que há evidências insuficientes de segurança e eficácia no tratamento de crianças para reduzir a morbimortalidade em adultos. Todavia, o consenso de 1992 não diz respeito às crianças de alto risco com história familiar de doença cardiovascular precoce e/ou hipercolesterolemia nos pais, as quais podem se beneficiar do tratamento medicamentoso em idade precoce. Além disso, o tratamento medicamentoso baseia-se apenas nos níveis de LDL-C e não leva em consideração outros fatores de risco para doença aterosclerótica.

O tratamento medicamentoso proposto pela I Diretriz de Prevenção da Aterosclerose na Infância e na Adolescência, embora considere como primeira escolha as estatinas, leva em consideração apenas o nível do LDL-C e não leva em consideração o nível de outras anormalidades, como diminuição do HDL-C, hipertrigliceridemia, tampouco outras alterações relacionadas com obesidade e $\mathrm{SM}^{14}$. Nesse sentido, faz-se necessário revisar os critérios para tratamento medicamentoso para prevenção de doença aterosclerótica em crianças e adolescentes de alto risco ${ }^{46}$.

McCrindle et al. ${ }^{2}$ recentemente estabeleceram novas recomendações para o início do tratamento medicamentoso da dislipidemia em crianças e adolescentes de alto risco. A avaliação do perfil lipídico deve ser realizada em crianças com história familiar e/ou naquelas com sobrepeso ou obesidade. Nestas últimas, devem ser avaliados outros componentes da SM, como resistência insulínica, diabetes tipo 2, hipertensão e obesidade central.

Os critérios para tratamento medicamentoso são os mesmos do consenso de 1992. A droga de primeira escolha é a estatina. Em alguns casos, pode ser considerado o início do tratamento antes dos 10 anos de idade. Crianças com anormalidades do perfil lipídico aliado à presença adicional de fatores de risco podem ter seu ponto de corte do nível sérico de LDL-C para iniciar terapia medicamentosa diminuído. São considerados fatores de risco: 
- história familiar marcante de doença ou evento cardiovascular precoce;

- $\quad$ associação de baixo nível de HDL-C, alto nível de triglicérides e partículas de LDL-C de pequeno tamanho;

- associação de sobrepeso ou obesidade e sinais de SM;

- presença de outra condição médica associada com aumento do risco de aterosclerose como diabetes, AIDS, lupus eritematoso sistêmico, transplante de órgão ou câncer;

- presença de hipertensão arterial;

- tabagismo assim como exposição passiva ao fumo;

- presença de outros marcadores elevação de homocisteína e proteína C-reativa.

\section{Drogas usadas no tratamento de dislipidemia}

\section{Resinas biliares}

As resinas biliares diminuem a absorção intestinal de ácidos biliares e aumentam a expressão de receptores hepáticos para o LDL-C, causando redução no colesterol sérico. Podem causar aumento nos níveis de triglicérides, interferem na absorção de vitaminas lipossolúveis e, frequentemente, causam efeitos colaterais gastrointestinais. A redução no nível sérico de LDL-C varia entre 13 e $20 \%$, e sua dose varia entre 2 e $12 \mathrm{~g} / \mathrm{dia}^{2,4,14}$. Embora o consenso de 1992 tenha o uso das resinas biliares como primeira escolha, na prática observa-se péssima palatabilidade e tolerância, contribuindo para sua baixa efetividade $e^{2,4,14}$.

\section{Estatinas}

As estatinas diminuem a síntese de colesterol por meio da enzima HGM-CoA redutase, limitando passo na síntese de colesterol. Essa diminuição do esterol celular faz com que o gene do receptor do LDL-C aumente o número de receptores de LDL-C e, consequentemente, diminua a circulação dessa lipoproteína ${ }^{2,4}$.

Os efeitos colaterais das estatinas incluem hepatotoxicidade com aumento das transaminases (geralmente transitório), miotoxicidade com mialgia e/ou raramente rabdomiólise e ainda teratogenicidade. Adolescentes do sexo feminino em uso de estatinas devem estar devidamente orientadas quanto aos métodos contraceptivos. Podem ocorrer ainda interações medicamentosas com aumento do risco de toxicidade (macrolídeos, antifúngicos, inibidores da protease, bloqueadores do canal de cálcio e ciclosporina e diminuição do nível sérico das estatinas, como rifampicina, barbitúricos e carbamazepina). Sua eficácia e segurança em crianças e em adolescentes são similares à encontrada em adultos ${ }^{2}$.

Atualmente existem quatro estatinas aprovadas pela Food and Drug Administration nos EUA para o uso clínico: sinvastatina, lovastatina, atorvastatina e pravastatina. A dose da sinvastatina é de 10 a $40 \mathrm{mg} / \mathrm{dia}$; a da lovastatina é de 10 a $40 \mathrm{mg} / \mathrm{dia}$; a da atorvastatina é de 10 a $20 \mathrm{mg} / \mathrm{dia}$; e a dose da pravastatina é de $20 \mathrm{mg} /$ dia para crianças com idade de 8 a 13 anos e de $40 \mathrm{mg} /$ dia para idade de 14 a 18 anos $^{4}$. A redução nos níveis de LDL-C varia entre 21 e $41 \%^{2}$. A rosuvastatina, uma nova estatina, mostrou-se eficaz e segura para o tratamento de hiperlipidemia em adultos com dislipidemia primária, mas estudos em criança ainda não foram realizados ${ }^{47-49}$.

\section{Ácido nicotínico}

O ácido nicotínico é uma vitamina solúvel com ação não totalmente conhecida, que reduz os níveis de triglicérides, LDL-C e aumenta os níveis de HDL-C. É raramente utilizado no tratamento da população pediátrica, pois tem grande incidência de efeitos colaterais como doença diarreica e outros mais graves como intolerância à glicose, miopatia, hiperuricemia e insuficiência hepática fulminante.

\section{Fibratos}

Os fibratos são medicamentos derivados do ácido fíbrico, com mecanismo de ação complexo, que age reduzindo os níveis de triglicérides e aumentando os níveis de HDL-C. Podem ainda causar diminuição nos níveis de LDL-C. A dose do benzofibrato varia de 10 a $20 \mathrm{mg} /$ dia e seus efeitos colaterais incluem: elevação das transaminases e creatinoquinases, miopatia e rabdomiólise, principalmente quando combinado ao uso de estatinas. Essa classe de drogas pode ser usada preferencialmente em crianças com elevação grave de triglicérides e com alto risco de desenvolverem pancreatite (triglicérides $\geq 400 \mathrm{mg} / \mathrm{dL}$ ) $2,4,14,50$.

\section{Inibidores da absorção do colesterol}

Inibidores específicos da absorção do colesterol são novos agentes que diminuem os níveis de lipídeos. A ezetimiba reduz o LDL-C em cerca de $20 \%$ e os triglicérides em $5 \%$; esses causam aumento discreto do HDL-C (1\%). É usada, preferencialmente, em conjunto com as estatinas em crianças com hiperlipidemia grave que não respondem à monoterapia com estatinas. Seu uso em crianças com idade superior a 10 anos já foi aprovado nos EUA para pacientes com hipercolesterolemia grave na dose de $10 \mathrm{mg} / \mathrm{dia}^{2,14}$.

\section{Tratamento medicamentoso com estatina}

A $\mathrm{AHA}^{2}$ desenvolveu manual para uso de estatinas em crianças, cujo resumo encontra-se na Tabela 2 . Suas recomendações incluem os critérios de seleção (idade e concentração sérica de LDL-C) do NCEP, podendo ser influenciado pela presença e magnitude de outros fatores de risco para doença cardiovascular, assim como a presença de xantomas cutâneos. É importante incluir os pais e familiares na decisão do tratamento. Em geral, o tratamento não deve ser iniciado antes dos 10 anos em meninos e antes da menarca nas meninas; deve-se aguardar o estágio puberal Tanner igual ou maior que II. Doença hepática, insuficiência renal, miopatia e gravidez constituem contraindicações para o tratamento medicamentoso com estatinas ${ }^{2,51}$.

Os pacientes em tratamento com estatinas devem ser monitorizados quanto ao crescimento (estatura, peso, índice de massa corporal), maturação sexual e desenvolvimento 
Tabela 2 - Indicações e monitoração do uso de estatinas em crianças e adolescentes com dislipidemias segundo $\mathrm{AHA}^{2}$

1) As estatinas são as drogas de primeira escolha. A decisão de qual estatina usar é de acordo com a experiência e/ou preferência do profissional.

2) Inicie com a menor dose uma vez ao dia, antes de dormir. Efetue dosagem sérica de creatinoquinase, ALT e AST.

3) Instrua o pacientes quanto aos efeitos colaterais, especialmente miopatias. Se ela estiver presente, avalie sua relação com atividade física, suspenda a medicação e efetue dosagem dos níveis séricos de creatinoquinase. $\mathrm{O}$ tratamento pode ser reconsiderado quando os sintomas e alterações laboratoriais desaparecerem.

4) Alerte as adolescentes quanto ao uso de medidas contraceptivas.

5) Alerte quanto às interações medicamentosas, especialmente com ciclosporina, fibratos, niacina, eritromicina, antifúngicos azólicos, nefazodone e inibidores da protease.

6) Após 4 semanas, monitorize a dosagem sérica de creatinoquinase, AST e ALT e compare-a com os níveis basais. Níveis séricos de creatinoquinase 10 vezes acima do limite superior devem ser investigados (considere o impacto da atividade física), assim como de ALT e AST 3 vezes acima do limite superior. A concentração mínima de LDL-C aceitável deve ser abaixo de $130 \mathrm{mg} / \mathrm{dL}$, e o ideal abaixo ou igual a 110 $\mathrm{mg} / \mathrm{dL}$.

7) Se a concentração de LDL-C desejável for atingida e não houver alterações laboratoriais, continue o tratamento e monitore os exames bioquímicos em 8 semanas e, posteriormente, em 3 meses.

8) Se existirem anormalidades laboratoriais ou sintomas, suspenda a droga temporariamente e repita os exames em 2 semanas. Se houver normalização dos parâmetros bioquímicos, a estatina pode ser reiniciada com monitoração.

9) Se a concentração de LDL-C não atingiu o objetivo, dobre a dose e repita os exames laboratoriais em 4 semanas. Continue aumentando a dose até o máximo recomendado até que o LDL-C atinja níveis desejados e, principalmente, se não houver evidência clínica ou laboratorial de toxicidade.

$\mathrm{AHA}=$ American Heart Association; ALT = alanino aminotransferase; $\mathrm{AST}$ = aspartato aminotransferase.

(estádio puberal de Tanner). Dosagens laboratoriais bioquímicas (creatinoquinase, alanino aminotransferase e aspartato aminotransferase) devem ser realizadas a cada 3 ou 6 meses. A dieta deve ser monitorizada, estimulando o baixo teor de lipídeo e deixando clara sua importância para o sucesso do tratamento. As crianças devem ser aconselhadas sobre outros fatores de risco como ganho de peso, tabagismo e sedentarismo ${ }^{2}$. Embora haja estudos que mostrem segurança e efetividade do tratamento com estatinas em crianças, a ausência de estudos em longo prazo faz com que estas recomendações devam ser usadas com critério.

\section{Considerações finais}

O papel do pediatra na prevenção e no tratamento da aterosclerose é fundamental. Deve-se ressaltar que a manutenção de um estilo de vida saudável, ainda na infância, é a base na prevenção da doença aterosclerótica e suas comorbidades que atingem a população cada vez em idade mais precoce.

As evidências de estudos com tratamento medicamentoso em crianças com dislipidemias primárias mostram segurança e eficácia similar às encontradas em adultos. Os consensos antigos apresentavam limitações, principalmente por não abordar a recente epidemia de sobrepeso e obesidade infantil. Essas novas recomendações incluem outros fatores de risco envolvidos na fisiopatologia da aterosclerose. Enfatiza-se que o tratamento medicamentoso só deve ser usado em crianças com anormalidades lipídicas de alto risco ou em outras condições de risco associadas que não responderem às mudanças dos hábitos de vida e não deve ser usado como primeira linha de tratamento.

\section{Conclusões}

Deve-se enfatizar que o tratamento medicamentoso só deve ser tentado em pacientes com anormalidades lipídicas graves ou com condições de alto risco após falha das modificações do hábito alimentar e estilo de vida ou quando essas alterações não estão diretamente relacionadas ao comportamento de vida do paciente. Cabe ao pediatra utilizar os conhecimentos científicos a fim de melhorar a qualidade de vida do seu paciente, utilizando sempre o binômio benefício/risco do paciente pediátrico a curto, médio e longo prazo. 


\section{Referências}

1. McMahan CA, Gidding SS, Malcom GT, Tracy RE, Strong JP, McGill HC Jr, et al. Pathobiological determinants of atherosclerosis in youth risk scores are associated with early and advanced atherosclerosis. Pediatrics. 2006;118:1447-55.

2. McCrindle BW, Urbina EM, Dennison BA, Jacobson MS, Steinberger J, Rocchini AP, et al.; American Heart Association Atherosclerosis, Hypertension, and Obesity in Youth Committee; American Heart Association Council of Cardiovascular Disease in the Young; American Heart Association Council on Cardiovascular Nursing. Drug therapy of high-risk lipid abnormalities in children and adolescents: a scientific statement from the American Heart Association Atherosclerosis, Hypertension, and Obesity in Youth Committee, Council of Cardiovascular Disease in the Young, with the Council on Cardiovascular Nursing. Circulation. 2007; 115:1948-67.

3. McGill HC Jr, McMahan CA. Starting earlier to prevent heart disease. JAMA. 2003;290:2320-2.

4. Belay B, Belamarich PF, Tom-Revzon C. The use of statins in pediatrics: knowledge base, limitations, and future directions. Pediatrics. 2007;119:370-80.

5. Freedman DS, Dietz WH, Srinivasan SR, Berenson GS. The relation of overweight to cardiovascular risk factors among children and adolescents: the Bogalusa Heart Study. Pediatrics. 1999;103:1175-82.

6. Raitakari OT, Juonala M, Kahönen M, Taittonen L, Laitinen T, Mäki-Torkko N, et al. Cardiovascular risk factors in childhood and carotid artery intima-media thickness in adulthood: the Cardiovascular Risk in Young Finns Study. JAMA. 2003; 290:2277-83.

7. Li S, Chen W, Srinivasan SR, Bond MG, Tang R, Urbina EM, et al. Childhood cardiovascular risk factors and carotid vascular changes in adulthood: the Bogalusa Heart Study. JAMA. 2003; 290:2271-6.

8. McMahan CA, Gidding SS, Fayad ZA, Zieske AW, Malcom GT, Tracy RE, et al. Risk scores predict atherosclerotic lesions in young people. Arch Intern Med. 2005;165:883-90.

9. Wiegman A, de Groot E, Hutten BA, Rodenburg J, Gort J, Bakker HD, et al. Arterial intima-media thickness in children heterozygous for familial hypercholesterolemia. Lancet. 2004; 363:369-70.

10. Wiegman A, Rodenburg J, de Jongh S, Defesche JC, Bakker HD, Kastelein Jj, et al. Family history and cardiovascular risk in familial hypercholesterolemia: data in more than 1000 children. Circulation. 2003;107:1473-8.

11. Ferreira AP, Oliveira CE, França NM. Metabolic syndrome and risk factors for cardiovascular disease in obese children: the relationship with insulin resistance (HOMA-IR). J Pediatr (Rio J). 2007; 83:21-6.

12. Dirisamer A, Hachemian N, Bucek RA, Wolf F, Reiter M, Widhalm $\mathrm{K}$. The effect of low-dose simvastatin in children with familial hypercholesterolaemia: a 1-year observation. Eur J Pediatr. 2003;162:421-5.

13. Giuliano IC, Caramelli B, Pellanda L, Duncan B, Mattos S, Fonseca FH. I Diretriz de Prevenção da Aterosclerose na Infância e na Adolescência. Arq Bras Cardiol. 2005:85 Supl 6:3-36.

14. American Academy of Pediatrics. National Cholesterol Education Program: Report of the Expert Panel on Blood Cholesterol Levels in Children and Adolescents. Pediatrics. 1992;89:525-84.
15. Jolliffe $\mathrm{CJ}$, Janssen I. Distribution of lipoproteins by age and gender in adolescents. Circulation. 2006;114:1056-62.

16. American Heart Association Nutrition Committee, Lichtenstein AH, Appel LJ, Brands M, Carnethon M, Daniels S, et al. Effect of docosahexaenoic acid on lipoprotein subclasses in hyperlipidemic children (the EARLY study). Circulation. 2006;114:82-96.

17. Engler MM, Engler MB, Malloy MJ, Paul SM, Kulkarni KR, Mietus-Snyder ML. Effect of docosahexaenoic acid on lipoprotein subclasses in hyperlipidemic children (the EARLY study). Am J Cardiol. 2005;95:869-71.

18. Sääkslahti A, Numminen $P$, Varstala V, Helenius $H$, Tammi A, Viikari J, et al. Physical activity as a preventive measure for coronary heart disease risk factors in early childhood. Scand J Med Sci Sports. 2004;14:143-9.

19. Kelley GA, Kelley KS. Aerobic exercise and lipids and lipoproteins in children and adolescents: a meta-analysis of randomized controlled trials. Atherosclerosis. 2007;191:447-53.

20. Gidding SS, Barton BA, Dorgan JA, Kimm SY, Kwiterovich PO, Lasser NL, et al. Higher self-reported physical activity is associated with lower systolic blood pressure: the Dietary Intervention Study in Childhood (DISC). Pediatrics. 2006; 118:2388-93.

21. Wiegman A, Hutten BA, de Groot E, Rodenburg J, Bakker HD, Büller HR, et al. Efficacy and safety of statin therapy in children with familial hypercholesterolemia: a randomized controlled trial. JAMA. 2004;292:331-7.

22. Hedman M, Matikainen T, Föhr A, Lappi M, Piippo S, Nuutinen M, et al. Efficacy and safety of pravastatin in children and adolescents with heterozygous familial hypercholesterolemia: a prospective clinical follow-up study. J Clin Endocrinol Metab. 2005; 90:1942-52.

23. Clauss SB, Holmes KW, Hopkins P, Stein E, Cho M, Tate A, et al. Efficacy and safety of lovastatin therapy in adolescent girls with heterozygous familial hypercholesterolemia. Pediatrics. 2005; 116:682-8.

24. Rodenburg J, Vissers MN, Wiegman A, van Trotsenburg AS, van der Graaf $A$, de Groot $E$, et al. Statin treatment in children with familial hypercholesterolemia: the younger, the better. Circulation. 2007;116:664-8.

25. Huang TT, Nansel TR, Belsheim AR, Morrison JA. Sensitivity, specificity, and predictive values of pediatric metabolic syndrome components in relation to adult metabolic syndrome: the Princeton LRC follow-up study. J Pediatr. 2008;152:185-90.

26. Chen W, Berenson GS. Metabolic syndrome: definition and prevalence in children. J Pediatr (Rio J). 2007;83:1-2.

27. Sinaiko A. Obesity, insulin resistance and the metabolic syndrome. J Pediatr (Rio J). 2007;83:3-4.

28. Cook S, Weitzman M, Auinger P, Nguyen M, Dietz WH. Prevalence of a metabolic syndrome phenotype in adolescents: findings from the third National Health and Nutrition Examination Survey, 1988-1994. Arch Pediatr Adolesc Med. 2003;157:821-7.

29. Brandão AP, Brandão AA, Nogueira AR, Suplicy $H$, Guimarães JI, de Oliveira JE. I Diretriz Brasileira de Diagnóstico e Tratamento da Síndrome Metabólica. Arq Bras Cardiol. 2005;84 Supl 1.

30. Weiss R, Dziura J, Burgert TS, Tamborlane WV, Taksali SE, Yeckel $\mathrm{CW}$, et al. Obesity and the Metabolic Syndrome in Children and Adolescents. N Engl J Med. 2004;350:2362-74.

31. Boyd GS, Koenigsberg J, Falkner B, Gidding S, Hassink S. Effect of obesity and high blood pressure on plasma lipid levels in children and adolescents. Pediatrics. 2005;116:442-6. 
32. Morrison JA, Friedman LA, Wang P, Glueck CJ. Metabolic syndrome in childhood predicts adult metabolic syndrome and type 2 diabetes mellitus 25 to 30 years later. J Pediatr. 2008; 152:201-6.

33. Lottenberg SA, Glezer A, Turatti LA. Metabolic syndrome: identifying the risk factors. J Pediatr (Rio J). 2007;83:S204-8.

34. Romaldini CC, Issler $\mathrm{H}$, Cardoso AL, Diament J, Forti N. Risk factors for atherosclerosis in children and adolescents with family history of premature coronary artery disease. J Pediatr (Rio J). 2004;80:135-40.

35. Sun SS, Grave GD, Siervogel RM, Pickoff AA, Arslanian SS, Daniels SR. Systolic blood pressure in childhood predicts hypertension and metabolic syndrome later in life. Pediatrics. 2007; 119:237-46.

36. Sun SS, Liang R, Huang TT, Daniels SR, Arslanian S, Liu K, et al. Childhood obesity predicts adult metabolic syndrome: the Fels Longitudinal Study. J Pediatr. 2008;152:191-200.

37. McCrindle BW, Ose L, Marais AD. Efficacy and safety of atorvastatin in children and adolescents with familial hypercholesterolemia or severe hyperlipidemia: a multicenter, randomized, placebo-controlled trial. J Pediatr. 2003;143:74-80.

38. Kavey RE, Allada V, Daniels SR, Hayman LL, McCrindle BW, Newburger JW, et al. Cardiovascular risk reduction in high-risk pediatric patients: a scientific statement from the American Heart Association Expert Panel on Population and Prevention Science; the Councils on Cardiovascular Disease in the Young, Epidemiology and Prevention, Nutrition, Physical Activity and Metabolism, High Blood Pressure Research, Cardiovascular Nursing, and the Kidney in Heart Disease; and the Interdisciplinary Working Group on Quality of Care and Outcomes Research: endorsed by the American Academy of Pediatrics. Circulation. 2006;114:2710-38.

39. American Academy of Pediatrics. Cardiovascular risk reduction in high-risk pediatric populations. Pediatrics. 2007;119:618-21.

40. Farley J, Gona P, Crain M, Cervia J, Oleske J, Seage G, et al. Prevalence of elevated cholesterol and associated risk factors among perinatally HIV-infected children (4-19 years old) in Pediatric AIDS Clinical Trials Group 219C. J Acquir Immune Defic Syndr. 2005;38:480-7.

41. Cheseaux JJ, Jotterand V, Aebi C, Gnehm H, Kind C, Nadal D, et al. Hyperlipidemia in HIV-infected children treated with protease inhibitors: relevance for cardiovascular diseases. J Acquir Immune Defic Syndr. 2002;30:288-93.

42. Tassiopoulos K, Williams PL, Seage GR 3rd, Crain M, Oleske J, Farley J. Association of Hypercholesterolemia Incidence With Antiretroviral Treatment, Including Protease Inhibitors, Among Perinatally HIV-Infected Children. J Acquir Immune Defic Syndr. 2008;47:607-14.
43. Dubé MP, Stein JH, Aberg JA, Fichtenbaum CJ, Gerber JG, Tashima $\mathrm{KT}$, et al. Guidelines for the evaluation and management of dyslipidemia in human immunodeficiency virus (HIV)-infected adults receiving antiretroviral therapy: recommendations of the HIV Medical Association of the Infectious Disease Society of America and the Adult AIDS Clinical Trials Group. Clin Infect Dis. 2003;37:613-27.

44. Bonnet $F$, Balestre $E$, Thiébaut $R$, Mercié $P$, Dupon $M$, Morlat $P$, et al. Fibrates or statins and lipid plasma levels in 245 patients treated with highly active antiretroviral therapy. Aquitaine Cohort, France, 1999-2001. HIV Med. 2004;5:133-9.

45. Calza L, Manfredi R, Chiodo F. Statins and fibrates for the treatment of hyperlipidaemia in HIV-infected patients receiving HAART. AIDS. 2003;17:851-9.

46. Gidding SS. New cholesterol guidelines for children? Circulation. 2006;114:989-91.

47. Shepherd J, Hunninghake DB, Stein EA, Kastelein JJ, Harris S, Pears J, et al. Safety of rosuvastatin. Am J Cardiol. 2004; 94:882-8.

48. Stein EA, Strutt K, Southworth H, Diggle PJ, Miller E; HeFH Study Group. Comparison of rosuvastatin versus atorvastatin in patients with heterozygous familial hypercholesterolemia. Am J Cardiol. 2003;92:1287-93.

49. Jones PH, Davidson MH, Stein EA, Bays HE, McKenney JM, Miller $\mathrm{E}$, et al. Comparison of the efficacy and safety of rosuvastatin versus atorvastatin, simvastatin, and pravastatin across doses (STELLAR* Trial). Am J Cardiol. 2003;92:152-60.

50. Hayman LL, Meininger JC, Daniels SR, McCrindle BW, Helden L, Ross J, et al. Primary prevention of cardiovascular disease in nursing practice: focus on children and youth: a scientific statement from the American Heart Association Committee on Atherosclerosis, Hypertension, and Obesity in Youth of the Council on Cardiovascular Disease in the Young, Council on Cardiovascular Nursing, Council on Epidemiology and Prevention, and Council on Nutrition, Physical Activity, and Metabolism. Circulation. 2007;116:334-57.

51. Stone NJ. Stopping Statins. Circulation. 2004;110:2280-2.

Correspondência:

Fernanda Luisa Ceragioli Oliveira

Disciplina de Nutrologia/Departamento de Pediatria

UNIFESP/EPM

Rua Loefgreen, 1647

CEP 04040-032 - São Paulo, SP

Tel.: (11) 5573.1246

E-mail: fernandalco.dped@epm.br 